\title{
O uso de recursos digitais no ensino de Radiologia Odontológica: uma revisão integrativa de literatura
}

\author{
Elza Bernardes Monier*; Denizar Vianna Araujo**; Ana Emilia Figueiredo de Oliveira***; Ana \\ Carolina Uruçu Rego Fernandes****; Luana Martins Cantanhede*****; Manuela Almeida \\ Montenegro Furtado*******
}

\author{
Doutoranda em Ciências Médicas, UERJ \\ Doutor em Saúde Coletiva UERJ \\ Doutora em Radiologia Odontológica, UNICAMP \\ Mestranda em Saúde do Adulto e da Criança, UFMA \\ Doutoranda em Odontologia, UFMA \\ Mestre em Biotecnologia, UFC
}

Recebido em 08/08/2017. Aprovado em 09/01/2018.

\begin{abstract}
RESUMO
Na Odontologia, diversos recursos vêm sendo adotados como estratégias de auxílio ao processo de ensino-aprendizagem na perspectiva de integrar a teoria com a prática. Este estudo objetivou levantar os principais recursos educacionais digitais na área de Radiologia Odontológica e discorrer sobre as características de aprendizagem destes recursos, de acordo com o modelo de Kirkpatrick. Realizouse revisão integrativa de trabalhos publicados no período de 2007 a 2017, nas bases de dados PubMed, Biblioteca Virtual em Saúde e Biblioteca Digital Brasileira de Teses e Dissertações, considerando os descritores: "computer-assisted instruction"; "dental education"; "educational technology"; "radiology". A amostra final foi composta por 13 artigos que atenderam aos critérios de inclusão e exclusão, em que foram identificados os seguintes recursos educacionais digitais: 3 módulos interativos baseado na Web; 3 objetos de aprendizagem digital; 2 cursos em plataforma virtual; 2 ferramentas digitais interativas; 1 software de visualização 3D; 1 simulador em radiologia oral e 1 sistema tecnológico de resposta ao aluno. A partir modelo de aprendizagem adotado, foram identificados os seguintes níveis nos trabalhos: satisfação dos participantes e/ou ganho de conhecimento dos participantes antes ou depois de uma intervenção ou ambos. A presente revisão de literatura identificou 7 tipos de recursos educacionais digitais utilizados em radiologia oral, que apresentaram atitudes positivas dos alunos em relação ao e-learning em todos os estudos, além de ganho significativo do conhecimento na maioria dos estudos.
\end{abstract}

Descritores: Instrução por Computador. Tecnologia Educacional. Odontologia. Radiologia.

\section{INTRODUÇÃO}

Os computadores tornaram-se parte integrante de nossas vidas pessoais $\mathrm{e}$ profissionais, e um resultado óbvio disso é a introdução de computadores e dispositivos eletrônicos pessoais nos domínios do ensino e da aprendizagem ${ }^{1}$. Do ponto de vista pedagógico, o que é descrito como instrução assistida por 
computador, aprendizagem assistida por computador ou e-learning consiste em uma vasta gama de metodologias de instrução e ferramentas para facilitar o aprendizado. Dentre os principais recursos tecnológicos de aplicação ao ensino odontológico, citam-se as aplicações de teleconferência, aprendizagem assistida por computador, simulações, plataformas de elearning, e avaliação baseada em computador ${ }^{2}$.

$\mathrm{Na}$ área da Odontologia, o emprego destas tecnologias no ensino de Radiologia Odontológica tem sido relatado na literatura. Esta especialidade está integrada com as demais áreas de da Odontologia como importante auxiliar no diagnóstico, planejamento e prognóstico das afecções bucais, tendo apresentado constante desenvolvimento nos últimos anos. Atualmente, imagens digitais podem ser visualizadas, gerenciadas, armazenadas e transmitidas por meio de redes de computadores ${ }^{3,4}$. Contudo, estudo recente afirma que o aprendizado eletrônico em Radiologia Odontológica ainda é bastante incomum ${ }^{5}$.

Existem relatos de que os computadores podem ser usados de forma interativa para melhorar a motivação e facilitar a consolidação do conhecimento. Também se entende que os ambientes digitais permitem aos professores apresentar conteúdos de forma visualmente efetiva, incentivando a participação dos estudantes ${ }^{6,7}$.

É importante, no entanto, que se compreenda a eficiência da aprendizagem alcançada pelo e-learning, a fim de esclarecer quando e como utilizar este método ${ }^{3}$. Kirkpatrick $^{8}$ criou um modelo de avaliação de treinamentos em quatro níveis: 1) reação, no qual se mede a satisfação e valor percebido do treinamento pelos participantes;

aprendizagem, que levanta o quanto os participantes podem mudar de atitude, ampliar seus conhecimentos e/ou habilidades; (3) comportamento, identifica o quanto os participantes mudaram seu comportamento em decorrência do que foi aprendido; e (4) resultados, que identifica os ganhos obtidos com o treinamento.

Assim sendo, esta revisão integrativa objetivou levantar os principais recursos educacionais digitais na área de Radiologia Odontológica e discorrer sobre as características de aprendizagem destes recursos, de acordo com o modelo de Kirkpatrick.

\section{METODOLOGIA}

Trata-se de revisão integrativa da literatura, que apresenta como vantagem a inclusão de múltiplos estudos com diferentes delineamentos de pesquisa9. Para tanto, foram utilizadas as seguintes perguntas norteadoras: (1) "Quais os principais recursos educacionais digitais na área de Radiologia Odontológica?" e (2) "Quais as características de aprendizagem (satisfação do aluno, resultado de aprendizagem, mudança de prática e resultado em saúde) foram avaliadas nos estudos que utilizam estes recursos?"

O levantamento da literatura foi realizado considerando as seguintes bases de dados: PubMed, Biblioteca Virtual em Saúde (BVS) e Biblioteca Digital Brasileira de Teses e Dissertações (BDTD). Foram adotados os descritores não controlados: "computer-assisted instruction"; "dental education"; "educational technology”; "radiology". A busca nas bases de dados foi realizada em abril de 2017, utilizando os operadores booleanos AND e OR. Tais termos permitem realizar combinações dos descritores utilizados na localização dos artigos, sendo AND uma combinação restritiva e OR uma combinação aditiva.

A análise dos estudos ocorreu em duas etapas: na primeira, após o cruzamento dos descritores, foi realizada a leitura dos títulos e resumos da amostra total, e separados os que 
estavam relacionados aos critérios de inclusão. $\mathrm{Na}$ segunda, os estudos pré-selecionados foram analisados na íntegra, quando foram utilizados os critérios de inclusão e exclusão. Estes critérios foram: estudos publicados na íntegra, nos idiomas inglês ou português; que utilizaram recurso educacional digital no ensino de Radiologia Odontológica; publicados entre 2007 e 2017. Foram excluídos os estudos que não apresentaram claramente em seus resultados algum nível de avaliação de aprendizagem do modelo de Kirkpatrick e trabalhos repetidos.

\section{RESULTADOS}

Na primeira etapa foram identificadas 181 publicações sobre a temática em questão. A figura 1 apresenta o diagrama do processo de identificação, seleção, elegibilidade e inclusão de estudos.
Na etapa 2, após a aplicação dos critérios de inclusão/exclusão, foram selecionados 13 trabalhos constituindo-se, assim, a amostra do estudo, cujos aspectos gerais estão descritos na tabela 1.

Dos treze trabalhos identificados, onze estavam no formato de artigo científico e dois no formato tese de doutorado, sendo dois no idioma português, e os demais em inglês. Todos tratavamse de trabalhos de pesquisa, realizados em centros de estudos ou universidades, tendo como amostras estudantes de diversos níveis e profissionais. Foram identificados sete tipos de recursos educacionais digitais: 3 módulo interativo baseado na Web; 3 objetos de aprendizagem digital; 2 cursos em plataforma virtual; 2 ferramentas digitais interativas; 1 software de visualização 3D; 1 simulador em radiologia oral e 1 sistema tecnológico de resposta ao aluno.

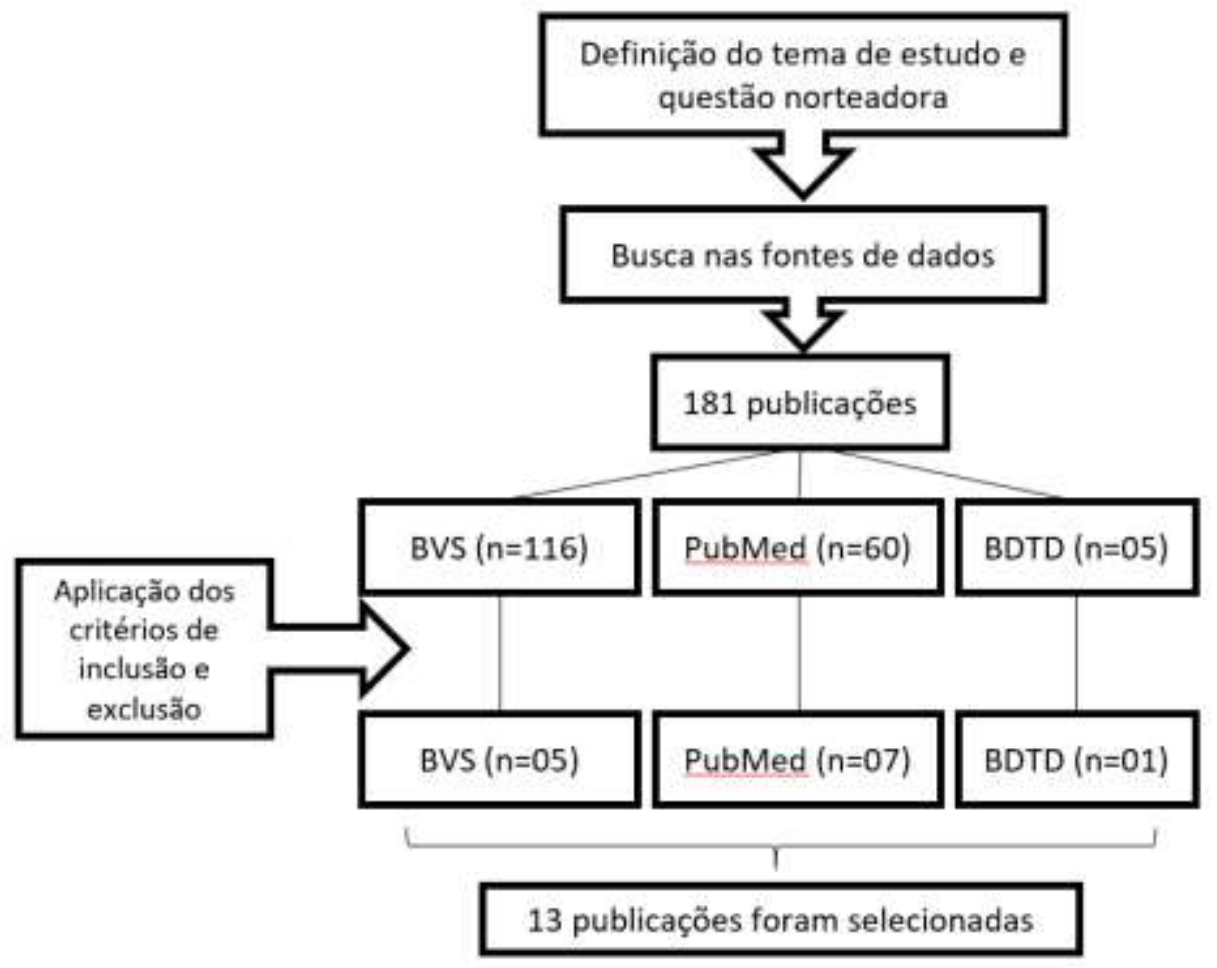

Figura 1. Fluxograma do processo de seleção de artigos que enfocam os recursos educacionais digitais usados na educação da radiologia odontológica entre os anos de 2007 a 2017 
Tabela 1. Características dos estudos selecionados

\begin{tabular}{|c|c|c|c|c|c|c|}
\hline Título & Autores & Ano & Recurso educacional & Objetivo(s) & Amostra & Tipo \\
\hline $\begin{array}{l}\text { 1. Interactive learning in oral and } \\
\text { maxillofacial radiology. }\end{array}$ & $\begin{array}{l}\text { Ramesh A, } \\
\text { Ganguly R. }\end{array}$ & 2016 & $\begin{array}{l}\text { Sistema tecnológico de resposta } \\
\text { ao aluno }\end{array}$ & $\begin{array}{l}\text { Avaliar correlação entre o desempenho em exames de } \\
\text { curso e questionários de autoavaliação }\end{array}$ & $\begin{array}{l}354 \text { estudantes de } \\
\text { Odontologia do } 2^{\circ} \text {. ano. }\end{array}$ & Artigo \\
\hline $\begin{array}{l}\text { 2. Evaluation of a digital learning object } \\
\text { (DLO) to support the learning process } \\
\text { in radiographic dental diagnosis. }\end{array}$ & Busanello FH, et al. & 2015 & $\begin{array}{l}\text { Objeto de aprendizagem digital } \\
\text { denominado Visual Basic } \\
\text { Application (VBA) }\end{array}$ & $\begin{array}{l}\text { Avaliar um objeto de aprendizagem digital, } \\
\text { desenvolvido para melhorar habilidades no diagnóstico } \\
\text { de alterações dentárias radiográficas. }\end{array}$ & $\begin{array}{l}62 \text { estudantes, divididos } \\
\text { em } 2 \text { grupos }\end{array}$ & Artigo \\
\hline $\begin{array}{l}\text { 3. Distance learning in dental radiology: } \\
\text { immediate impact of the } \\
\text { implementation }\end{array}$ & $\begin{array}{l}\text { Cruz AD, Costa JJ, } \\
\text { Almeida SM. }\end{array}$ & 2014 & Plataforma Moodle & $\begin{array}{l}\text { Avaliar o impacto imediato da aprendizagem de anato- } \\
\text { mia radiográfica periapical após substituição do méto- } \\
\text { do de ensino em sala de aula pela educação a distância }\end{array}$ & $\begin{array}{l}122 \text { estudantes divididos } \\
\text { em } 2 \text { grupos }\end{array}$ & Artigo \\
\hline $\begin{array}{l}\text { 4. Evaluation of a blended learning course } \\
\text { for teaching oral radiology to } \\
\text { undergraduate dental students. }\end{array}$ & Kavadella A, et al. & 2012 & $\begin{array}{l}\text { Curso combinado (instruções } \\
\text { presenciais e online) }\end{array}$ & $\begin{array}{l}\text { Desenvolver, implementar e avaliar a eficácia } \\
\text { educacional (derivada do desempenho dos alunos e } \\
\text { respostas aos questionários) de um curso combinado. }\end{array}$ & $\begin{array}{l}47 \text { estudantes divididos } \\
\text { em dois grupos }\end{array}$ & Artigo \\
\hline $\begin{array}{l}\text { 5. Digital interactive learning of oral } \\
\text { radiographic anatomy. }\end{array}$ & $\begin{array}{l}\text { Vuchkova J, } \\
\text { Maybury T, Farah } \\
\text { CS. }\end{array}$ & 2012 & Ferramenta digital interativa & $\begin{array}{l}\text { Avaliar uma ferramenta digital interativa comparada a } \\
\text { um livro de radiologia convencional em anatomia } \\
\text { radiográfica. }\end{array}$ & $\begin{array}{l}88 \text { estudantes ( } 64 \text { do } 2^{\circ} \text {. } \\
\text { ano e } 24 \text { do } 5^{\circ} \text {. ano), } \\
\text { divididos em } 2 \text { grupos }\end{array}$ & Artigo \\
\hline $\begin{array}{l}\text { 6. Método de ensino-aprendizagem no } \\
\text { diagnóstico radiográfico das } \\
\text { anormalidades ósseas dos maxilares }\end{array}$ & Costa E. & 2012 & $\begin{array}{l}\text { Ferramenta colaborativa virtual } \\
\text { (DRAMA) }\end{array}$ & $\begin{array}{l}\text { Avaliar a Ferramenta colaborativa virtual (DRAMA) } \\
\text { em complemento à proposta metodológica no ensino } \\
\text { presencial. }\end{array}$ & $\begin{array}{l}88 \text { estudantes, divididos } \\
\text { em } 2 \text { grupos (teste e } \\
\text { controle) }\end{array}$ & Tese \\
\hline $\begin{array}{l}\text { 7. Desenvolvimento e teste de um objeto } \\
\text { digital de aprendizagem para } \\
\text { interpretação das imagens por } \\
\text { ressonância magnética da articulação } \\
\text { temporomandibular }\end{array}$ & Arús NA. & 2012 & $\begin{array}{l}\text { Objeto Digital de Aprendizagem } \\
\text { (ODA) para interpretação de } \\
\text { Ressonância Magnética da } \\
\text { Articulação Temporomandibular }\end{array}$ & $\begin{array}{l}\text { Efetuar avaliação entre aprendizagem interativa } \\
\text { (através de um ODA) e aprendizagem convencional }\end{array}$ & $\begin{array}{l}29 \text { estudantes divididos } \\
\text { em } 2 \text { grupos }\end{array}$ & Tese \\
\hline $\begin{array}{l}\text { 8. Testing the educational potential of 3D } \\
\text { visualization software in oral } \\
\text { radiographic interpretation. }\end{array}$ & $\begin{array}{c}\text { Vuchkova J, } \\
\text { Maybury TS, Farah } \\
\text { CS. }\end{array}$ & 2011 & $\begin{array}{l}\text { Software de visualização 3D } \\
\text { versus livro didático }\end{array}$ & $\begin{array}{l}\text { Realizar avaliação entre software de visualização 3D a } \\
\text { partir de imagens radiográficas 2D e livro didático }\end{array}$ & $\begin{array}{l}59 \text { estudantes do } 4^{\circ} \text { ano, } \\
\text { divididos em } 2 \text { grupos }\end{array}$ & Artigo \\
\hline $\begin{array}{l}\text { 9. Dental student skill retention eight } \\
\text { months after simulator-supported } \\
\text { training in oral radiology. }\end{array}$ & $\begin{array}{l}\text { Nilsson TA, } \\
\text { Hedman LR, } \\
\text { Ahlqvist JB. }\end{array}$ & 2011 & Simulador & $\begin{array}{l}\text { Investigar os efeitos a longo prazo na habilidade para } \\
\text { interpretar informações espaciais em radiografias após } \\
\text { treinamento convencional e simulador. }\end{array}$ & $\begin{array}{l}45 \text { estudantes do } 7^{\circ} \text { e } 9^{\circ} \\
\text { semestres, divididos em } \\
2 \text { grupos }\end{array}$ & Artigo \\
\hline $\begin{array}{l}\text { 10. Introduction of e-learning in dental } \\
\text { radiology reveals significantly } \\
\text { improved results in final examination. }\end{array}$ & Meckfessel S et al. & 2011 & Curso baseado na web & $\begin{array}{l}\text { Avaliar das percepções dos alunos que utilizaram } \\
\text { materiais didáticos baseado na web, bem como o efeito } \\
\text { sobre seus resultados nos exames finais }\end{array}$ & $\begin{array}{l}228 \text { estudantes divididos } \\
\text { em } 4 \text { grupos }\end{array}$ & Artigo \\
\hline $\begin{array}{l}\text { 11. Web-based training method for } \\
\text { interpretation of dental images. }\end{array}$ & Wu M et al. & 2010 & $\begin{array}{l}\text { Ferramenta baseada em } \\
\text { computador para um programa } \\
\text { educacional }\end{array}$ & $\begin{array}{l}\text { Fornecer ferramentas únicas baseadas em computador } \\
\text { para um programa educacional para melhorar as } \\
\text { habilidades de interpretação de radiografias }\end{array}$ & $\begin{array}{l}15 \text { participantes de uma } \\
\text { conferência, sendo a } \\
\text { maioria professores }\end{array}$ & Artigo \\
\hline $\begin{array}{l}\text { 12. Implementing e-learning in a } \\
\text { radiological science course in dental } \\
\text { education: a short-term longitudinal } \\
\text { study. }\end{array}$ & $\begin{array}{l}\text { Tan PL, Hay DB, } \\
\text { Whaites E. }\end{array}$ & 2009 & Módulos e-learning & $\begin{array}{l}\text { Avaliar comparativamente os diversos modos de } \\
\text { aprendizagem (face-a-face e e-learning, apenas face-a- } \\
\text { face e apenas e-learning) }\end{array}$ & 140 estudantes & Artigo \\
\hline $\begin{array}{l}\text { 13. Evaluation of the radiographic } \\
\text { cephalometry learning process by a } \\
\text { learning virtual object. }\end{array}$ & Silveira HLD, et al. & 2007 & $\begin{array}{l}\text { Objeto virtual de aprendizagem } \\
\text { (OVA) Ceph Learning }\end{array}$ & $\begin{array}{l}\text { Testar de OVA Ceph Learning, verificando a melhoria } \\
\text { de desempenho dos estudantes }\end{array}$ & $\begin{array}{l}40 \text { estudantes, divididos } \\
\text { em } 2 \text { grupos }\end{array}$ & Artigo \\
\hline
\end{tabular}


Quanto à avaliação da aprendizagem, foram encontrados os seguintes resultados (tabela 2). Nível 1: Satisfação do aluno, avaliado por meio de análise qualitativa em 12 dos 13 estudos selecionados, utilizando questionários. Em todos os trabalhos foram identificadas atitudes positivas em relação aos recursos testados. Nível 2: resultados de ganho de conhecimento, analisado em 12 trabalhos, cuja aferição foi feita utilizando-se de diversos recursos avaliativos, tais como questionários de escolha múltipla, imagens radiográficas para interpretar, testes de prática, dentre outros. Os resultados variaram: 8 estudos relataram ganho significativo de conhecimento ${ }^{1,4,6,10-14}$; um não encontrou diferença significativa ${ }^{15}$; em um estudo grupo que não utilizou o recurso educacional apresentou melhor desempenho ${ }^{16}$; e um trabalho observou resultados inferiores de conhecimento após a intervenção em comparação aos escores iniciais ${ }^{17}$. Ainda, um trabalho apresentou pontuação elevada no teste de habilidade visual, porém as pontuações na avaliação da interpretação radiográfica não sofreram mudanças após a intervenção ${ }^{7}$.

Não foram identificados estudos que abordaram os níveis de aprendizagem 3 (Comportamento) e 4 (Resultado), pela dificuldade de sua aferição na educação em saúde.

\section{DISCUSSÃO}

A integração de ferramentas tecnológicas na vida cotidiana também aumentou a disponibilidade e o uso desses recursos na educação ${ }^{1}$. Nas áreas do ensino médico e odontológico, a formação com técnicas auxiliadas por computadores tem sido cada vez mais utilizada ${ }^{3,7}$.

Entende-se como aprendizagem multimídia todas as formas de aprendizagem que utilizam meios digitais para apresentar e distribuir materiais de aprendizagem $^{6}$. Foram identificados os seguintes recursos educacionais digitais: sistemas/módulos interativos baseados na web $6,13,18$, objetos de aprendizagem digital ${ }^{10,14,16}$, cursos em plataforma virtual $^{11,15}$, ferramentas digitais interativas ${ }^{4,7}$, software para visualização $3 \mathrm{D}^{17}$; simulador ${ }^{12} \mathrm{e}$ sistema tecnológico de resposta ao aluno, denominado Learning Catalytics ${ }^{\mathrm{TM}}$ (LC) (Pearson, Nova York, NY, EUA) ${ }^{1}$.

$\mathrm{O}$ sucesso de qualquer forma de ensino e determinação de resultados de aprendizagem específicos foram geralmente medidos durante o processo de avaliação. Muitos trabalhos não avaliam os resultados da adoção do ensino eletrônico, o que não permite identificar se a metodologia adotada pode ou não reduzir a qualidade dos resultados de aprendizagem $^{13}$. Por este motivo, realizou-se a avaliação de aprendizagem dos recursos educacionais digitais.

Sabe-se da evidência do aumento do uso ferramentas online para a educação odontológica $^{19,20}$. Os trabalhos investigados reforçam este relato, apontando, também, para o ganho do conhecimento com o uso do e-learning ${ }^{5,10}$. Este achado está de acordo com autores ${ }^{21,22}$ que afirmam que a aprendizagem assistida por computador é tão eficaz quanto os métodos tradicionais.

Diversos pontos positivos sobre o uso de recursos educacionais digitais na educação odontológica têm sido relatados na literatura ${ }^{23}$. Tal afirmativa dialoga com os resultados encontrados nos trabalhos estudados, destacando o fato dos recursos poderem ser utilizados repetidamente, sem restrições de tempo ou loca ${ }^{15,13}$, flexibilidade do ensino $^{13}$, bem como a facilitação do desenvolvimento de habilidades na resolução de problemas antes do efetivo contato com pacientes propiciados pelo uso de tais ferramentas ${ }^{3}$.

No entanto, também há relatos de críticas e restrições ao e-learning, como a necessidade da simplificação da navegação dos programas, da presença de mais exercícios de autoavaliação ${ }^{6}$, de capacitação docente para utilizar o e-learning ${ }^{3}$, além do entendimento de que esses recursos não podem trazer sozinhos uma mudança no ensino. Em vez disso, o método de ensino-aprendizagem existente precisa ser fortalecido ${ }^{5}$. 
Tabela 2. Resultados de avaliação de aprendizagem nos estudos selecionados segundo o modelo de Kirkpatrick

\begin{tabular}{|c|c|c|c|c|}
\hline \multirow{3}{*}{ 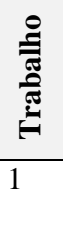 } & \multicolumn{2}{|c|}{ Resultados de avaliação da aprendizagem, segundo modelo de Kirkpatrick } & \multicolumn{2}{|c|}{ Recursos avaliativos utilizados } \\
\hline & Nível 1 & Nível 2 & Nível 1 & Nível 2 \\
\hline & $\begin{array}{l}\text { Feedback positivo sobre o uso de LC no curso } \\
\text { de radiologia }\end{array}$ & $\begin{array}{l}\text { Correlação positiva, porém fraca, encontrada entre } 0 \\
\text { desempenho dos alunos em exames e em testes de classe }\end{array}$ & Declarações de avaliação & Questionário online \\
\hline 2 & Avaliação positiva, de pontuação $(81,6$ a 90,5$)$ & Melhor desempenho do grupo teste & Escala de Usabilidade de Sistema (SUS) & Testes teórico e prático \\
\hline 3 & Nível de satisfação positivo & Sem diferença significativa entre as notas dos grupos & Questionário & Prova \\
\hline 4 & $\begin{array}{l}\text { Atitudes relação aos elementos do aprendizado } \\
\text { combinado foram muito positivas }\end{array}$ & $\begin{array}{l}\text { Grupo combinado apresentou desempenho significativamente } \\
\text { melhor do que o convencional no teste pós-curso. }\end{array}$ & $\begin{array}{l}\text { Questionários dicotômicos, questões } \\
\text { de escala aberta e de cinco pontos } \\
\text { (Likert) }\end{array}$ & $\begin{array}{l}\text { Questões de certo/errado, múltipla escolha, } \\
\text { perguntas e imagens radiográficas para interpretar }\end{array}$ \\
\hline 5 & $\begin{array}{l}\text { Maioria preferiu a ferramenta digital, todos a } \\
\text { consideraram de fácil uso, a maioria sentiu-se } \\
\text { mais confiante aprendendo com a mesma }\end{array}$ & $\begin{array}{l}\text { Teste de habilidade visual-espacial: todos os participantes } \\
\text { obtiveram pontuação elevada; avaliação da interpretação } \\
\text { radiográfica: pontuações não mudaram após a intervenção }\end{array}$ & Questionário usando escala Likert & $\begin{array}{l}\text { Testes de múltipla escolha / testes de rotações } \\
\text { mentais (MRT) }\end{array}$ \\
\hline 6 & $\begin{array}{l}94,1 \% \text { dos alunos consideram válida ou muito } \\
\text { válida a contribuição da metodologia }\end{array}$ & $\begin{array}{l}\text { Melhor desempenho do grupo que utilizou a ferramenta em } \\
\text { contraponto ao grupo que não fez o uso da mesma. }\end{array}$ & Questionário de múltipla escolha & $\begin{array}{l}\text { Questionários de escolha múltipla; imagens } \\
\text { radiográficas para interpretar, testes escritos }\end{array}$ \\
\hline 7 & $\begin{array}{l}\text { Avaliação positiva da usabilidade (SUS) } \\
\text { (efetividade, eficiência e satisfação), escore } 89\end{array}$ & $\begin{array}{l}\text { Os } 2 \text { grupos melhoraram o desempenho; grupo controle } \\
\text { apresentou melhor desempenho. }\end{array}$ & Escala de Usabilidade de Sistema (SUS) & Prova objetiva \\
\hline 8 & $\begin{array}{l}\text { Maioria preferiu usar a visualização 3D do que } \\
\text { o livro. Todos indicaram a necessidade de uso } \\
\text { combinado da visualização 3D com o livro }\end{array}$ & $\begin{array}{l}\text { Após a intervenção, o escore obtido foi menor do que na pré- } \\
\text { intervenção, sugerindo que o uso do software de visualização } \\
\text { 3D não melhorou a performance dos estudantes }\end{array}$ & $\begin{array}{l}\text { Métodos qualitativos, utilizando um } \\
\text { levantamento estruturado de escala } \\
\text { Likert }\end{array}$ & Teste de Rotação Mental (MRT) \\
\hline 9 & - & $\begin{array}{l}\text { Habilidade de interpretar relações espaciais após o uso de } \\
\text { simulador foi melhor } 8 \text { meses após o treinamento }\end{array}$ & - & Teste de Rotação Mental (MRT) \\
\hline 10 & $\begin{array}{l}\text { Maioria com atitude positiva em relação ao } e \text { - } \\
\text { learning }\end{array}$ & Redução de reprovação nos exames finais & Questionário usando escala Likert & Prova de múltipla escolha \\
\hline 11 & $\begin{array}{l}\text { Avaliação muito favorável, maior pontuação } \\
\text { para "aprendizado" e menor para "design" }\end{array}$ & - & Questionário & - \\
\hline 12 & $\begin{array}{l}\text { O e-learning foi bem avaliado., porém, o } \\
\text { ensino presencial teve melhor índice na } \\
\text { avaliação }\end{array}$ & $\begin{array}{l}\text { Melhores resultados foram alcançados pelo grupo de alunos } \\
\text { com aprendizagem mista (face-a-face e e-learning) }\end{array}$ & $\begin{array}{l}\text { Pesquisa de feedback (questionário on } \\
\text { line) }\end{array}$ & Exame de múltipla escolha \\
\hline 13 & Aprovação de aspectos de usabilidade & $\begin{array}{l}\text { No primeiro pós-teste, o grupo B, no qual os alunos usaram o } \\
\text { objeto, apresentou maior pontuação média e marcos mais } \\
\text { corretamente identificados em comparação com o grupo A, } \\
\text { mas essa diferença não foi estatisticamente significante. No } \\
\text { entanto, no segundo pós-teste } 15 \text { dias depois, houve diferenças } \\
\text { estatisticamente significativas entre os grupos, com o grupo B } \\
\text { apresentando melhores resultados do que o grupo A. }\end{array}$ & Escala de usabilidade de sistema (SUS) & Questionário de múltipla escolha \\
\hline
\end{tabular}


Tais considerações reforçam a condição precípua do professor de moderador do conhecimento $^{3}$ e, assim, os recursos para elearning sejam utilizados como um complementares na educação em Radiologia Odontológica ${ }^{4,11,12,14,16 .}$

É importante ressaltar que na Odontologia, especificamente para a área da Radiologia, o papel do computador é claramente mais decisivo do que para outras disciplinas, pois a área de diagnóstico por imagem faz uso, de forma mais rotineira, das imagens digitais. $\mathrm{Na}$ educação radiológica, o aluno deve ser exposto a uma grande quantidade de informações visuais e a assimilação dessas imagens é essencial na formação de sua capacidade diagnóstica, constituindo, portanto, em um importante auxílio no ensino da radiologia3,6.

Fica, então, a reflexão de que aprendizagem não pode ser embasada apenas pela introdução de uma ferramenta tecnológica por si só, mas o impacto que as ferramentas tecnológicas podem ter na habilitação de um ambiente de aprendizagem interativo é impressionante ${ }^{1,3} \mathrm{e}$ atitudes receptivas dos alunos podem impulsionar o e-learning para cursos em educação superior, como a Odontologia, especialmente no campo da Radiologia Odontológica $^{13}$.

\section{CONCLUSÃO}

Foram descritos 7 tipos recursos educacionais digitais utilizados no ensino da Radiologia Odontológica Constatou-se que a maioria dos estudos sobre e-learning nesta área publicados nos últimos 10 anos baseia a avaliação no grau de satisfação dos participantes e/ou ganho de conhecimento depois da intervenção. Em todos os estudos foram identificadas atitudes positivas dos estudantes em relação ao e-learning, além de ganho significativo do conhecimento na maioria das vezes. Não foram encontradas pesquisas que demonstrassem mudanças de desempenho na prática clínica ou no resultado obtido com o paciente.

\section{ABSTRACT \\ The use of digital resources in the teaching of Dental Radiology: an integrative literature review}

In dentistry, several resources have been adopted as strategies to aid the teaching-learning process in order to integrate theory and practice. This study aims to raise the main digital educational resources in the area of Dental Radiology and discuss the learning characteristics of these resources, according to the Kirkpatrick model. An integrative review of papers published between 2007 and 2017 was carried out in the databases PubMed, Virtual Health Library and Brazilian Digital Library of Theses and Dissertations, considering the descriptors: "computer-assisted instruction"; dental education"; "educational technology"; "radiology". The final sample consisted of 13 articles that met the inclusion and exclusion criteria, in which the following digital educational resources were identified: 03 interactive module based on the Web; 03 digital learning objects; 02 virtual platform courses; 02 interactive digital tools; $013 \mathrm{D}$ visualization software; 01 simulator in oral radiology and 01 technological system of response to the student. From the adopted learning model, the following levels were identified in the study: participants' satisfaction and / or gain of knowledge of the participants before or after an intervention or both. The present literature review identified 7 types of digital educational resources used in oral radiology, which presented positive attitudes of students in relation to e-learning in all studies, in addition to a significant gain in knowledge in most of the studies.

Descriptors: Computer-assisted Instruction. Educational Technology. Dentistry. Radiology.

\section{REFERÊNCIAS}

1. Ramesh A, Ganguly R. Interactive learning 
in oral and maxillofacial radiology. Imaging Sci Dent. 2016;46:211-6.

2. Mattheos N, Stefanovic N, Apse P, Attstrom R, Buchanan J, Brown P, et al. Potential of information technology in dental education. Eur J Dent Educ. 2008;12:85-91.

3. Santos GNM, Leite AF, Figueiredo PT de S, Pimentel NM, Flores-Mir C, de Melo NS, et al. Effectiveness of e-learning in oral radiology education: a systematic review. J Dent Educ. 2016;80:1126-39.

4. Costa E. Método de ensino-aprendizagem no diagnóstico radiográfico das anormalidades ósseas dos maxilares [tese] Florianópolis: Faculdade de Engenharia e Gestão do Conhecimento. Universidade Federal de Santa Catarina; 2012.

5. Chang H-J, Symkhampha K, Huh K-H, Yi W-J, Heo M-S, Lee S-S, et al. The development of a learning management system for dental radiology education: A technical report. Imaging Sci Dent. 2017;47:51-55.

6. Meckfessel S, Stühmer C, Bormann KH, Kupka T, Behrends M, Matthies H, et al. Introduction of e-learning in dental radiology reveals significantly improved results in final examination. J CranioMaxillofacial Surg. 2011;39:40-8.

7. Vuchkova J, Maybury T, Farah CS. Digital interactive learning of oral radiographic anatomy. Eur J Dent Educ. 2012;16:79-87.

8. Maloney S, Haas R, Keating JL, Molloy E, Jolly B, Sims J, et al. Effectiveness of Webbased versus face-to-face delivery of education in prescription of falls-prevention exercise to health professionals: randomized trial. J Med Internet Res. 2011;13(4):e116. DOI: 10.2196/jmir.1680.

9. Mendes KDS, Silveira RC de CP, Galvão CM. Revisão integrativa: método de pesquisa para a incorporação de evidências na saúde e na enfermagem. Texto \& Contexto Enferm.2008;17:758-64.

10. Busanello FH, da Silveira PF, Liedke GS Arús NA, Vizzotto MB, Silveira HE, et al. Evaluation of a digital learning object (DLO) to support the learning process in radiographic dental diagnosis. Eur J Dent Educ. 2015;19:222-8.

11. Kavadella A, Tsiklakis K, Vougiouklakis G, Lionarakis A. Evaluation of a blended learning course for teaching oral radiology to undergraduate dental students. Eur J Dent Educ. 2012;16:88-95.

12. Nilsson TA, Hedman LR, Ahlqvist JB. Dental student skill retention eight months after simulator-supported training in oral radiology. J Dent Educ. 2011;75:679-84.

13. Tan P, Hay DB, Whaites E. Science Course in Dental Education: A Short-Term Longitudinal Study. J Dent Educ. 2009;73:1202-12.

14. Silveira HLD, Gomes MJ, Silveira HED, Dalla-Bona RR. Evaluation of the radiographic cephalometry learning process by a learning virtual object. Am J Orthod Dentofac Orthop. 2009;136:134-8.

15. Cruz AD, Costa JJ, Almeida SM. Distance learning in dental radiology: immediate impact of the implementation. Brazilian Dent Sci. 2014;17:90-97.

16. Arús NA. Desenvolvimento e teste de um objeto digital de aprendizagem para interpretação das imagens por ressonância magnética da articulação temporomandibular [tese]. Porto Alegre: Faculdade de Odontologia, Universidade Federal do Rio Grande do Sul; 2012.

17. Vuchkova J, Maybury TS, Farah CS. Testing the educational potential of 3D visualization software in oral radiographic interpretation. J Dent Educ. 2011;75:1417-25. R

18. Wu M, Zhang X, Koenig L, Lynch J, Wirtz 
T, Mao E, et al. Web-based training method for interpretation of dental images. J Digit Imaging. 2010;23:493-500.

19. Pahinis K, Stokes CW, Walsh TF, Cannavina G. Evaluating a blended-learning course taught to different groups of learners in a dental school. J Dent Educ. 2007;71:269-78.

20. Salajan, F., Mount G. University of Toronto's dental school shows "new teeth": moving towards online instruction. J Dent Educ. 2008;72:532-42.

21. Al-jewair TS, Azarpazhooh A, Suri S, Shah PS. Computer-Assisted Learning in Orthodontic Education: A Systematic Review and Meta-Analysis. J Dent Educ.
2009;73:730-9.

22. Al-Jewair TS, Qutub AF, Malkhassian G, Dempster LJ. A systematic review of computer-assisted learning in endodontics education. J Dent Educ. 2010;74:601-11.

23. Schleyer TK, Johnson LA. Evaluation of educational software. J Dent Educ. 2003;67:1221-8.

Correspondência para:

Elza Bernardes Monier

e-mail: bernardes.elza@gmail.com

Av. da Paz, bloco A, ap 204, Cond Rei Salomão V, 65072-570 Parque Shalon - São Luís/MA 